\title{
Cancer Progenitor Cells: The Result of an Epigenetic Event?
}

\author{
KAROLINA LAPINSKA ${ }^{1}$, GABRIELA FARIA ${ }^{2}$, SANDRA MCGONAGLE $^{2,3}$, \\ KATE MORGAN MACUMBER ${ }^{2}$, SARAH HEERBOTH ${ }^{4}$ and SIBAJI SARKAR SA, $^{2,5}$ \\ ${ }^{1}$ Quinnipiac University School of Medicine, North Haven, CT, U.S.A.; \\ ${ }^{2}$ Mass Bay Community College, Wellesley, MA, U.S.A.; \\ ${ }^{3}$ Quincy College, Quincy, MA, U.S.A.; \\ ${ }^{4}$ Vanderbilt University School of Medicine, Nashville, TN, U.S.A.; \\ ${ }^{5}$ Roxbury Community College, Boston, MA, U.S.A.
}

\begin{abstract}
The concept of cancer stem cells was proposed in the late 1990s. Although initially the idea seemed controversial, the existence of cancer stem cells is now well established. However, the process leading to the formation of cancer stem cells is still not clear and thus requires further research. This article discusses epigenetic events that possibly produce cancer progenitor cells from predisposed cells by the influence of their environment. Every somatic cell possesses an epigenetic signature in terms of histone modifications and DNA methylation, which are obtained during lineage-specific differentiation of pluripotent stem cells, which is specific to that particular tissue. We call this signature an epigenetic switch. The epigenetic switch is not fixed. Our epigenome alters with aging. However, depending on the predisposition of the cells of a particular tissue and their microenvironment, the balance of the switch (histone modifications and the DNA methylation) may be tilted to immortality in a few cells, which generates cancer progenitor cells.
\end{abstract}

Stem cells are pluripotent cells that are formed during embryogenesis after generation of blastocysts (1-6). These pluripotent stem cells are capable of growing and differentiating into specialized cells. The management of

This article is freely accessible online.

Correspondence to: Sibaji Sarkar, Ph.D., Division of Biotechnology, Quincy College, 1250 Hancock St, Quincy, MA 02169, U.S.A. Tel: +1 6175660524, e-mail: ssarkarmmb@gmail.com

Key Words: Stem cells, cancer stem cells, cancer progenitor cells, epigenetics, methylation, histone modifications, signaling, cancer drug resistance, cancer combination therapy, review. stem cell generation and the lineage-specific specialized cell and tissue formation are tightly regulated. This process is repeated with fidelity during embryogenesis and includes many reversible processes, which we term as 'on and off switches' (Sarkar et al., unpublished; 1-6). From the start of fertilization until the generation of specific tissues, each step is governed by conserved histone modifications and differential DNA methylation. Any particular grouping of histone modifications in combination with a set of DNA methylation is what we have defined as an 'epigenetic switch' (7-9). The generation of pluripotent stem cells occurs in the later stages of embryogenesis, and involves wellorchestrated hormonal regulation, effects of stromal cells (such as their micro-environment), and intracellular signaling (1-6). The generation of cancer stem cells and their role in formation and development of cancer have drawn huge attention since their discovery around the late 1990s.

A significant amount of literature is available on almost every type of cancer stem cell, covering their isolation, characteristics, culture, growth, ability to form tumors in animal models and their possible utilization in therapy. In this review, we confine ourselves to the role of epigenetics in the formation of cancer stem cells/cancer progenitor cells. We also compare the current paradigm with our hypothesis, which states that alteration in epigenetic balance (combination of histone modifications and 5'-C-phosphateG-3 (CpG) DNA methylation) provides the necessary switch for the formation of cancer progenitor cells.

\section{Cancer Progenitor Cells: Epigenetic Regulation}

Pluripotent stem cells are special in that they can both grow and differentiate at the same time. This quality is not present in somatic cells. Usually when somatic cells grow they do not differentiate much. Somehow cancer stem cells resemble adult 
stem cells. Depletion of histone 1 ( $\mathrm{H} 1$, all three variants) impairs the differentiation of pluripotent stem cells. It also impairs DNA methylation and changes histone marks at the promoter region of octamer-binding transcription factor (OCT). These results suggest that epigenetic mechanisms repress pluripotent genes to allow differentiation, a feature that is caused by a depletion of methylation of DNA (10). Thus these findings support our hypothesis that epigenetic alterations regulate growth and differentiation of pluripotent stem cells.

The classic example of the generation of induced pluripotent stem cells (iPS) was shown in skin cells by introduction of three transcription factors: OCT4, sexdetermining region Y-box 2 (SOX2) and homeoboxprotein NANOG (NANOG), which showed for the first time that the terminal differentiation of tissues at the end of embryogenesis is not irreversible (11). As we discussed before, embryogenesis is a tightly regulated epigenetic event, and thus it makes sense that for a particular tissue, such as skin, all the genes necessary for the structure and function of these cells are active and others, although intact, have been silenced by epigenetic mechanisms in these cells. Indeed, it his been observed that these master transcription factors, which can revert terminally differentiated skin cells to their pluripotent state, are tightly regulated by epigenetic mechanisms (12-14). This reversibility raises the possibility that adverse alteration in histone modifications and CpG DNA methylation could tilt the balance of these cells towards uncontrolled growth but lacking pluripotency and differentiation. Such a scenario will produce cancer stem cells/cancer progenitor cells. iPS cells are pluripotent, although not exactly identical to embryonic stem cells. Similarly, cancer stem cells (as titled in literature) are not precisely the same as normal stem cells. Thus, we prefer to distinguish them by the name cancer progenitor cells (15). If the cells are predisposed to other genetic alterations and imbalances, the possibility of cancer progenitor cell formation will increase.

Similarly to the generation of pluripotent stem cells, cancer progenitor cells also need signaling mechanisms to help produce them. These signaling mechanisms involve stromal cells around the tissues, cell surface receptors, hormones, growth factors, and alterations in intracellular signaling. Three of the major signaling pathways that are implicated in cancer progenitor cell formation are wingless family of genes (WNT), notch, and hedgehog (16). All three pathways are epigenetically regulated. We previously elaborated on the role of signaling in carcinogenesis $(12,17-20)$, which we will not include here and instead provide the link between epigenetic modulators and signaling molecules. For example, extracellular signal-regulated kinases (ERK) and v-ask murine thymoma viral oncogene homolog 1 (AKT) signaling pathways are two major regulators of cell growth and inhibition of apoptosis. Recent studies have shown that both of these pathways regulate DNA methylation $(12,17-20)$. It is interesting to mention here that this regulation is a reversible process, similar to a feedback loop. It has been observed that signaling processes regulate methylation and conversely epigenetic changes, such as methylation, also regulate signaling mechanisms. One of the major examples of this is the regulation of insulin-like growth factor 2 (IGF2), which plays a significant role in embryogenesis (21). Additionally, ERK signaling regulates DNA methyltransferase 1 (DNMT1) enzymes and thus regulates methylation (19). In a systems biology study, we further observed that DNMT1 is selectively and allosterically activated around the genes that are highly methylated in cancer cells (22).

As we mentioned before, tissue-specific gene expression, which we believe is regulated by a specific epigenetic switch, produces specific cell types where an epigenetic mark should be maintained within the adult cells of the tissues. This particular switch should be different between tissue types (Figure 1). Figure 1a is a simplistic portrayal of part of the epigenetic signature of various tissue types. A chromosome from any tissue, designated as Chromosome A' in Figure 1a, possesses $\mathrm{CpG}$ methylation on DNA at different locations. The examples of kidney, lung, and liver are provided here, although the same is true for other tissues. If we believe that the epigenetic switch is different but conserved in every tissue type, alteration of a particular switch will cause an imbalance in the gene expression in the cells of one type of tissue, which is distinct for these cells. Another parameter of the epigenetic switch is due to histone modifications that vary in different tissues. These histone modifications occur depending on where and when methylation, phosphorylation, ubiquitination, and somulation occur. For example, the levels of H3K27me3 and $\mathrm{H} 3 \mathrm{~K} 9 \mathrm{me} 3$ (inhibitors of gene expression), and $\mathrm{H} 3 \mathrm{~K} 4 \mathrm{me} 3$ (activator of gene expression) may not be similar in lung and liver. When methylation patterns on chromosomes change in the cells of a particular tissue, as shown in Figure 1b (see difference in methylation pattern in the chromosome marked 'Normal' and in the chromosome marked 'Cancer'), the binding of these histone marks will change at the particular DNA location (see Figure 2). In the chromosome marked 'Cancer', the pattern of gene expression will therefore be altered from that in the chromosome marked 'Normal'. However, this alteration will remain confined to this particular tissue and will result in differential gene expression in these particular tissue cells, which will produce cancer progenitor cells specific to this type of tissue. These imbalances could simultaneously suppress tumor-suppressor genes and express growth-promoting genes. As these changes are dependent on the intracellular and extracellular milieu, they cannot be as rapid as those caused by mutations. We previously hypothesized that mutations occurring in predisposed cells regulate how fast cancer or tumor will progress (7-9, 12, 17-19, 22, 23). Thus indeed, cancer progenitor cell formation is a slow process. 

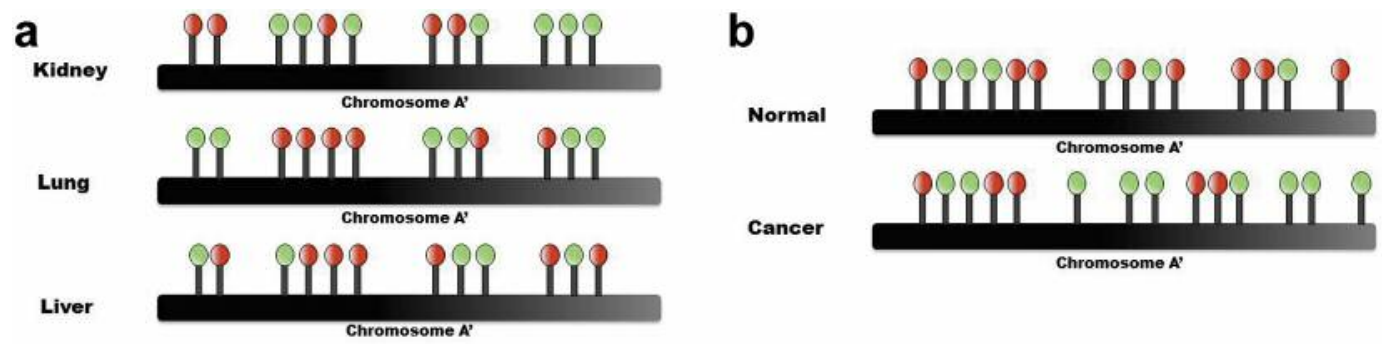

Figure 1. Differential methylation patterns of 5'-C-phosphate- $G-3(C p G)$ residues in a hypothetical chromosome A' in the kidney, lung and liver (a) and in normal and cancer cells of the same tissue (b). The black/gray line denotes the chromosome. Green balls are unmethylated CpG residues; red balls are methylated $C p G$ residues. It can be seen that methylation patterns of the same chromosome in the cells of different tissues are different (a). The total amount of methylation is lower in cancer cells compared to normal cells (b). This differential behavior possibly favors growth, promoting gene expression and suppression of tumor-suppressor genes.

Promoter methylation is known to silence tumorsuppressor genes. Studies from several laboratories have shown that interaction of enhancers with promoters is essential for gene transcription $(24,25)$. Some of these enhancers, which regulate tissue-specific gene expression, are called super-enhancers $(24,26)$. The methylation of super-enhancers regulates their function. These functions are dependent on the binding of H3K27me3 to unmethylated sites, creating a zone of inhibition involving CCCTCbinding factor (CTCF). Methylation disrupts the association of CTCF with DNA, which allows gene transcription. Alternatively, H3K9me3 recruits DNMT1 at the site of DNA methylation. In addition, $\mathrm{H} 3 \mathrm{~K} 4 \mathrm{me} 3$ interacts at hypomethylated promoter sites to increase gene transcription. Surprisingly, it was recently observed that methylation in the coding region in these genes can also involve binding of the modified histones. To add final control to these mechanisms, short RNAs and lncRNAs produced from noncoding regions also interact with the promoter region to control transcription, which is evident in imprinting. The best example of this is the regulation of IGF2 and IGF2 receptor $(21,27)$.

A classic work further determined that the generation of super-enhancers on oncogenes and other types of genes is important in tumor pathogenesis (24). H3K27ac ChIP-Seq analysis identified super-enhancers and their associated genes in 18 human cancer cells. These results suggested that genes associated with super-enhancers could be selective markers for specific types of cancer of specific tissue types. Myelocytomatosis oncogene (MYC) is one of these oncogenes. These super-enhancers are not present around such genes in normal cells. The authors suggested that one of the mechanisms by which super-enhancers are formed is tandem repeats of DNA segments $(24,26)$. We previously postulated that tandem repeats of DNA or gene segment are also possibly regulated by epigenetic mechanisms (23).

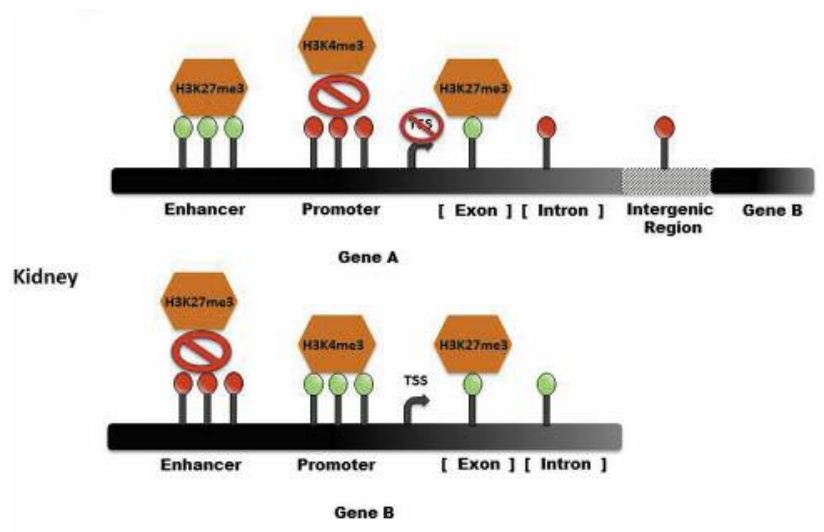

Figure 2. Differential binding of $H 3 K 27 m e 3$ and $H 3 K 4 m e 3$ at different locations in two hypothetical genes $A$ and $B$ in the kidney: The black/gray line denotes the chromosome. The green balls are unmethylated 5'-C-phosphate-G-3 (CpG) residues; red balls are methylated $C p G$ residues. The stop sign on transcription start site (TSS) signifies inhibition of transcription. The stop sign on $C p G$ residues signifies inhibition of binding. Differential binding of these histone markers at methylated (red) or unmethylated (green) sites on DNA regulates the outcome of collective gene expression in some selected predisposed cells of a tissue, which will determine whether they will become cancer progenitor cells. It is a slow, time-consuming process.

There are two clonal hypotheses of formation of cancer progenitor cells. The first is that one type of cancer progenitor cell further differentiates into diverse types of cells. The second hypothesis mentions that cancer progenitor cells simultaneously grow and differentiate. Current research shows that contrary to embryonic stem cells, which can grow and differentiate at the same time, cancer progenitor cells grow but do not differentiate until they enter the state of metastasis $(17,18)$. This is a very interesting point. This observation suggests that cancer progenitor cells under the 
control of a fixed epigenetic switch are altered for rapid uncontrolled growth, but do not revert to the pluripotent stage and thus cannot differentiate into other lineages. When a few cells are directed to form pluripotent stem cells of a particular lineage, they are allowed to grow, although they do not differentiate any more. Recent studies have shown that super-enhancers epigenetically regulate this process $(24$, 26). It makes sense to rationalize that epigenetic alterations of these tissue specific cancer progenitor cells allow uncontrolled growth, keeping the differentiation markers silenced. Although we observed that differentiation does occur at the later stages of tumor development during metastasis, this differentiation does not cause reversion to pluripotency. Conversely, this differentiation is conditional and only allows the process of epithelial to mesenchymal transition (EMT) and, after distant traveling and during localization to another site, mesenchymal to epithelial transition (MET) $(17,18)$. As these processes are reversible, we argue that they are epigenetically regulated and cannot possibly be mutagenic changes, which are unidirectional.

An interesting scenario in the case of solid tumors is that they are extremely heterogeneous, containing different types of mutations within the same type of tumor. This finding further puzzles researchers since isolated cancer progenitor cells from different tumor samples of similar tissue origin have differential cell-surface markers. Cancer progenitor cells of different tissue origins are usually characterized by specific cell-surface markers, which are used to isolate them $(28,29)$. The presence of $\mathrm{CD} 44^{+}, \mathrm{CD} 133^{+}, \mathrm{CD} 24^{+}$, and other markers are usually common. In leukemia progenitor cells, expression of CD47 helps them escape killing by macrophages. In addition, several other specific markers are present, according to the origin of tumors $(28,29)$. It was believed that the generation of cancer progenitor cells was dependent on a series of mutations (30). The problem with this theory is that the incorporation of isolated cancer progenitor cells in an animal model does not always produce tumors. Furthermore, a niche, such as an interactive microenvironment (stromal cells), is necessary to induce this process $(17,18)$. Other investigators implicated a role of feeder cells in the maintenance and growth of human embryonic stem cells (31). These results suggest that the generation of cancer progenitor cells is dependent more on epigenetic alterations. For example, it is known that mutation in ten-eleven translocation methylcytosine dioxygenase (TET) causes leukemia, although TET regulates methylation processes and can change the balance of its epigenetic switch (30).

As mutations can occur randomly, some authors believed that formation of cancer progenitor cells, cancer progression, and metastasis are random processes (32). We do not agree with this conclusion. As we described before, differential signaling processes are involved in cancer progenitor cell formation, it is possible that a few of the pathways could be activated epigenetically in a subset of cells of similar tissues, while other pathways will be activated in other subsets, resulting in differential expression of cell-surface markers. This event may result in cancer progenitor cells of same tissue origin with slightly different surface markers because the microenvironment of each cancer progenitor cell may differ. Even in this scenario, the epigenetic alterations of DNA methylation and differential binding of histone markers to DNA could make cells immortal (Figure 2). Figure 2 is a simplistic description of this process. In the hypothetically portrayed genes (Gene A and Gene B) of the kidney, enhancer, promoter, exons, introns, and intergenic regions are differentially methylated. Gene A favors binding of $\mathrm{H} 3 \mathrm{~K} 27 \mathrm{me} 3$ at the enhancer location (hypomethylated $\mathrm{CpG}$ residues), but does not bind $\mathrm{H} 3 \mathrm{~K} 4 \mathrm{me} 3$ at the promotor region (hypermethylated $\mathrm{CpG}$ residues) and inhibits transcription. Gene B does not bind to $\mathrm{H} 3 \mathrm{~K} 27 \mathrm{me} 3$ since its enhancer region is methylated, but allows binding of $\mathrm{H} 3 \mathrm{~K} 4 \mathrm{me} 3$ to its hypomethylated promoter region and thus transcription is activated. Figure 2 depicts that gene expression will be altered depending on where methylation occurs in and around the gene. We may imagine a wide variety of methylation patterns are to be expected in the same genes in different tissues. Depending on the predisposed genetic conditions and their surrounding, the methylation pattern will change further. This is usually a slow process as these modifications accumulate over time. This hypothesis is supported by the fact that a few of the cells around a tumor turn into cancer progenitor cells and are not killed by traditional therapies $(33,37,40)$.

Cancer progenitor cells are immortal. Like growing embryonic cells, cancer progenitor cells also evade killing by the normal immune system. In addition, these cancer progenitor cells also need to reduce their apoptotic mechanisms, an after-event of DNA damage. One study has shown that in human embryonic stem (hES) cells, proapoptotic BCL2-associated X protein (BAX) is present in the Golgi apparatus. During DNA damage or in the presence of chromosomal abnormalities, when these defects cannot be rectified $\mathrm{BAX}$ is translocated to the mitochondria by a p53dependent mechanism to induce apoptosis of the cells to prevent formation of faulty pluripotent stem cells (34). Cancer progenitor cells evade apoptosis by unique mechanisms. In some of the cancer progenitor cells, p53 is either mutated or is not present. In addition to these alterations, methylation and other epigenetic changes silence many cell-cycle inhibitors and tumor-suppressor genes to prevent apoptosis $(7-9,12,17-19,22,23)$.

\section{Cancer Progenitor Cells and Therapy}

Elucidation of the process of formation of cancer progenitor cells will produce an outstanding framework for treating cancer, reducing relapse, and killing drug-resistant cancer cells. Current therapies, which target specific molecules, as well as therapies that are not targeted, do reduce the tumor 
cell population and, depending on the cancer type, can achieve remission. However, in most cases the cancer relapses. It has been well studied in the case of leukemia, where acute lymphocytic leukemia (produced by a mutation in DNMT3A) can be treated to remission (35), although patients usually experience relapse after a few years. One study showed that higher methylation levels were observed in the cells of such patients and were maintained indefinitely, even after the remission (36). We believe that even after cancer is in remission, the presence of high methylation levels continues to lead to the generation of more cancer progenitor cells and helps produce drug-resistant cancer cells (7-9, 12, 17-19, 22, 23, 37-39). Therefore, we hypothesize that the best chemotherapy will be a combination of an epigenetic drug with standard therapies (7-9, 12, 17-19, 22, 23, 37-40). Current studies support this notion as combinations using epigenetic drugs kill cancer stem cells and sensitize drugresistant and other types of cancer cells (20,37-42).

\section{Conclusion}

In short, although complex and seemingly diverse, the production and maintenance of cancer progenitor cells should be an organized process, like epigenetically regulated embryogenesis. Further acquisition of mutations or inherited mutations makes this process faster or slower and provides diversity as observed in heterogeneous tumor populations. It remains to be discovered how differential epigenetic modifications are often confined to one particular tissue type to generate cancer progenitor cells of that particular type. We believe that this epigenetic process will depend on the status of pre-disposed cells, which includes their genetic and various chromosomal abnormalities in the particular tissue, as well as their microenvironment.

\section{Conflicts of Interest}

The Authors have no disclosure of conflict of interest in regard to this study.

\section{References}

1 Li E: Chromatin modification and epigenetic reprogramming in mammalian development. Nat Rev Genet 3: 662-673, 2002.

2 Messerschmidt DM, Knowles BB and Solter D: DNA methylation dynamics during epigenetic reprogramming in the germline and preimplantation embryos. Genes Dev 28: 812-828, 2014.

3 Haaf T: Methylation dynamics in the early mammalian embryo: implications of genome reprogramming defects for development. Curr Top Microbiol Immunol 310: 13-22, 2006.

4 Chotalia M, Smallwood SA, Ruf N, Dawson C, Lucifero D, Frontera M, James K, Dean W and Kelsey G: Transcription is required for establishment of germline methylation marks at imprinted genes. Genes Dev 23: 105-117, 2009.
5 Morgan HD, Santos F, Green K, Dean W and Reik W: Epigenetic reprogramming in mammals. Hum Mol Genet 14: R47-58, 2005.

6 Vastenhouw NL and Schier AF: Bivalent histone modifications in early embryogenesis. Curr Opin Cell Biol 24: 374-386, 2012.

7 Byler S, Goldgar S, Heerboth S, Leary M, Housman G, Moulton K and Sarkar S: Genetic and epigenetic aspects of breast cancer progression and therapy. Anticancer Res 34(3): 1071-1077, 2014.

8 Byler S and Sarkar S: Do epigenetic drug treatments hold the key to killing cancer progenitor cells? Epigenomics 6(2): 161-165, 2014.

9 Longacre M, Snyder NA, Housman G, Leary M, Lapinska K, Heerboth S, Willbanks A and Sarkar S: A comparative analysis of genetic and epigenetic events of breast and ovarian cancer related to tumorigenesis. IJMS 17(5): 759, 2016.

10 Zhang Y, Cooke M, Panjwani S, Cao K, Krauth B, Ho P, Medrzycki M, Berhe D, Pan C, McDevitt T and Fan Y: Histone H1 depletion impairs embryonic stem cell. PloS Genet 8(5): e1002691, 2012.

11 Takahashi K and Yamanaka S: Induction of pluripotent stem cells from mouse embryonic and adult fibroblast cultures by defined factors. Cell 126(4): 663-676, 2006.

12 Doi A, Park I-H, Wen B, Murakami P, Aryee MJ, Irizarry R, Herb B, Ladd-Acosta C, Rho J, Loewer S, Miller J, Schlaeger T, Daley GQ and Feinberg AP: Differential methylation of tissue- and cancer-specific $\mathrm{CpG}$ island shores distinguishes human induced pluripotent stem cells, embryonic stem cells and fibroblasts. Nat Genet 41(12): 1350-1353, 2009.

13 Kim K, Doi A, Wen B, Ng K, Zhao R, Cahan P, Kim J, Aryee MJ, Ji H, Ehrich L, Yabuuchi A, Takeuchi A, Cunniff KC, Hongguang H, Mckinney-Freeman S, Naveiras O, Yoon TJ, Irizarry RA, Jung N, Seita J, Hanna J, Murakami P, Jaenisch R, Weissleder R, Orkin SH, Weissman IL, Feinberg AP and Daley GQ: Epigenetic memory in induced pluripotent stem cells. Nat Genet 467: 285-290, 2010.

14 Papp B and Plath K: Epigenetics of reprogramming to induced pluripotency. Cell 152(6): 1324-1343, 2013.

15 Sarkar S, Goldgar S, Byler S, Rosenthal S and Heerboth S: Demethylation and re-expression of epigenetically silenced tumor-suppressor genes: sensitization of cancer cells by combination therapy. Epigenomics 5(1): 87-94, 2013.

16 Toh T, Lim J, Chow E: Epigenetics in cancer stem cells. Mol Cancer 16: 29, 2017.

17 Sarkar S, Horn G, Moulton K, Oza A, Byler S, Kokolus S and Longacre M: Cancer development, progression, and therapy: an epigenetic overview. IJMS 14: 21087-21113, 2013.

18 Heerboth S, Housman G, Leary M, Longacre M, Byler S, Lapinska K, Willbanks A and Sarkar S: EMT and tumor metastasis. Clin Transl Med 4: 6, 2015.

19 Sarkar S, Abujamra AL, Loew JE, Forman LW, Perrine SP and Faller DV: Histone deacetylase inhibitors reverse $\mathrm{CpG}$ methylation by regulating DNMT1 through ERK signaling. Anticancer Res 31(9): 2723-2732, 2011.

20 Lapinska K, Housman G, Byler S, Heerboth S, Willbanks A, Oza A and Sarkar S: The effects of histone deacetylase inhibitor and calpain inhibitor combination therapies on ovarian cancer cells. Anticancer Res 36(11): 5731-5742, 2016.

21 Bell AC and Felsenfeld G: Methylation of a CTC-dependent boundary controls imprinted expression of the Igf2 gene. Nature 405: 482-485, 2000. 
22 Samorodnitsky E, Ghosh E, Mazumder S and Sarkar S: Methylation by DNMT1 is more efficient in chronic lymphocytic leukemia cells than in normal cells. J Proteomics Bioinformatics S10: 004, 2014.

23 Willbanks A, Leary M, Greenshields M, Tyminski C, Heerboth S, Lapinska K, Haskins $\mathrm{K}$ and Sarkar S: The evolution of epigenetics: from prokaryotes to humans and its biological consequences. Gen and Epigenet 8: 25-36, 2016.

24 Whyte WA, Orlando DA, Hnisz D, Abraham BJ, Lin CY, Kagey MH, Rahl PB, Lee TI and Young RA: Master transcription factors and mediator establish super-enhancers at key cell identity genes. Cell 153: 307-319, 2013.

25 Hanssen LLP, Kassouf MT, Oudelaar AM, Biggs D, Downes DJ, Gosden M, Sharpe JA Sloane-Stanley JA, Hughes JR, Davies B and Higgs DR: Tissue-specific CTCF-cohesin-mediated chromatin architecture delimits enhancer interactions and function in vivo. Nature 19: 952-961, 2017.

26 Hnisz D, Abraham BJ, Lee TI, Lau A, Saint-Andre V, Sigova AA, Hoke HA and Young RA: Super-enhancers in the control of cell identity and disease. Cell 155: 934-947, 2013.

27 Marcho D, Bevilaqua A, Tremblay KD and Mager J: Tissuespecific regulation of Igf2r/Airn imprinting during gastrulation. Epigenetics Chromatin 8: 10, 2015.

28 Qiang L, Yang Y, Ma YJ, Chen FH, Zhang LB, Liu W, Qi Q, Lu $\mathrm{N}$, Tao L, Wang XT, You QD and Guo QL: Isolation and characterization of cancer stem-like cells in human glioblastoma cell lines. Cancer Lett 279: 13-21, 2009.

29 Dragu D, Necuka L, Bleotu C, Diaconu C and ChivuEconomescu M: Therapies targeting cancer stem cells: current trends and future challenges. World J Stem Cells 7(9): 1185$1201,2015$.

30 Weissman I: Evolution of normal and neoplastic tissue stem cells: progress after Robert Hooke. Philos Trans R Soc Lond B Biol Sci 370: 1680, 2014

31 Desai N, Rampbhia P and Gishto A: Human embryonic stem cell cultivation: historical perspective and evolution of xeno-free culture systems. Reprod Biol Endocrinol 13: 9, 2015.

32 Tomasetti C and Vogelstein B: Variation in cancer risk among tissues can be explained by the number of stem cell division. Science 347: 78-81, 2015

33 Gasch C, Ffrench B, O'leary JJ and Gallagher MF: Catching moving targets: cancer stem cell hierarchies, therapy-resistance \& considerations for clinical intervention. Mol Cancer 16: 43, 2017.
34 Dumitru R, Gama V, Fagan M, Bower J, Swahari V, Pevny L and Deshmukh M: Human embryonic stem cells have constitutively active Bax at the Golgi and are primed to undergo rapid apoptosis. Molecular Cell 46: 573-583, 2012.

35 Yang L, Rau R and Goodell MA: DNMT3A in haematological malignancies. Nat Rev Cancer 15: 152-165, 2015.

36 Agrawal S, Unterberg M, Koschmieder S, zur Stadt U, Brunnberg U, Verbeek W, Buchner T, Berdel WE, Serve H and Nuller-Tidow C: DNA methylation of tumor suppressor genes in clinical remission predicts the relapse risk in acute myeloid leukemia. Cancer Res 67: 1370-1377, 2007.

37 Housman G, Byler S, Heerboth S, Lapinska K, Longacre M, Snyder N and Sarkar S: Drug resistance in cancer: an overview. Cancers 6(3): 1769-1792, 2014.

38 Heerboth S, Lapinska K, Snyder N, Leary M, Rollinson S and Sarkar S: Use of epigenetic drugs in disease: an overview. Genet Epigenet 6(9): 9-19, 2014.

39 Sarkar S, Longacre M, Tatur N, Heerboth S and Lapinska K: Histone deactylases (HDACs): function, mechanism and inhibition. In: Encyclopedia of Analytical Chemistry, John Wiley \& Sons, Ltd pp. 1-9, 2014.

40 Mataga MA, Rosenthal S, Heerboth S, Devalapalli A, Kokolus S, Evans LR, Longacre M, Housman G and Sarkar S: Antibreast cancer effects of histone deacetylase inhibitors and calpain inhibitor. Anticancer Res 32(7): 2523-2529, 2012.

41 Pathania R, Ramachandran S, Mariappan G, Thakur P, Shi H, Choi JH, Manicassamy S, Kolhe R, Prasad PD, Sharma S, Lokeshwar BL, Ganapathy V, Thangaraju M: Combined inhibition of DNMT and HDAC blocks the tumorigenicity of cancer stem-like cells and attenuates mammary tumor growth. Cancer Res 76(11): 3224-3235, 2016.

42 Cacan E, Ali MW, Boyd NH, Hooks SB and Greer SF: Inhibition of HDAC1 and DNMT1 modulate RGS10 expression and decrease ovarian cancer chemoresistance. PLoS One 9: 1, 2014.

Received September 5, 2017

Revised November 13, 2017

Accepted November 15, 2017 